\title{
GENERALIZATIONS OF $M$-GROUPS
}

\author{
YAKOV BERKOVICH
}

(Communicated by Ronald Solomon)

\begin{abstract}
In this note we prove some generalizations of Taketa's theorem on solvability of $M$-groups.
\end{abstract}

Let $\operatorname{Irr}(G)$ denote the set of all complex irreducible characters of a finite group $G$ (only finite groups are considered in this note), $\operatorname{Irr}_{1}(G)$ the set of all nonlinear characters in $\operatorname{Irr}(G), \operatorname{Lin}(G)$ the set of all linear characters of $G$, and $\operatorname{Irr}(\tau)$ the set of the irreducible constituents of a character $\tau$. A character $\chi \in \operatorname{Irr}(G)$ is said to be monomial if there exist $H \leq G$ and $\lambda \in \operatorname{Lin}(H)$ such that $\chi=\lambda^{G}$. A group $G$ is said to be an $M$-group if all its irreducible characters are monomial. Taketa ([Hu], Satz 5.18.6(b)) has proved that $M$ groups are solvable. It is natural to suppose that a group $G$ is solvable if the set of its monomial irreducible characters is large. As a corollary of our considerations one obtains that a group $G$ is solvable if all characters of the set $\{\chi \in \operatorname{Irr}(G) \mid \chi(1)<b(G)\}$ are monomial (here $b(G)$ is the maximal degree of irreducible characters of $G$ ). Further on if each irreducible character of $G$ is induced from a character of degree at most 2 , then $G$ is solvable (Theorem 7).

In the sequel $S$ denotes a nonempty set of simple groups. A group $G$ is said to be an $S$-group if it is a tower of groups from $S$. We consider the group $G=\{1\}$ as an $S$-group. A character $\chi \in \operatorname{Irr}(G)$ is said to be $S$-monomial if there exist $H \leq G$ and $\lambda \in \operatorname{Irr}(H)$ such that $\chi=\lambda^{G}$ and $H / \operatorname{ker} \lambda$ is an $S$-group. The set of all $S$-monomial characters of $G$ is denoted by $\operatorname{Irr}_{S}(G)$.

Lemma 1. Let $N>\{1\}$ be a normal subgroup of a group $G$. If

$$
|\operatorname{Irr}(G)-\operatorname{Irr}(G / N)| \leq \mathbf{3},
$$

then $N$ is solvable.

Proof. Let $M \subseteq G$. Denote by $k_{G}(M)$ the number of conjugacy classes of $G$ having nonempty intersections with $M$. In particular $k(G)=k_{G}(G)$ is the class number of $G$. It is known that $G$ is solvable if $k(G) \leq 4$. So if $N$ is nonsolvable, then $N<G$. Since $k(G)=|\operatorname{Irr}(G)|$, by hypothesis

$$
k(G / N)+k_{G}(N)-1 \leq k(G) \leq k(G / N)+3
$$

Received by the editors February 10, 1994.

1991 Mathematics Subject Classification. Primary 20C15.

This research was supported in part by the Ministry of Science and Technology and the Ministry of Absorption of Israel. 
and $k_{G}(N) \leq 4$. In the sequel we suppose that $N$ is nonsolvable. By Burnside's $\{p, q\}$-theorem one has $k_{G}(N) \geq 4$. Thus, $k_{G}(N)=4$. Let

$$
c d(N)=\{\varphi(1) \mid \varphi \in \operatorname{Irr}(N)\}=\left\{1, d_{1}, \ldots, d_{s}\right\}, \quad 1<d_{1}<\cdots<d_{s} .
$$

Take $\varphi_{i} \in \operatorname{Irr}(N)$ with $\varphi_{i}(1)=d_{i}, i=1, \ldots, s$. It follows from Clifford's Theorem that $\left\langle\varphi_{i}^{G}, \varphi_{j}^{G}\right\rangle=0$ for $i \neq j$, so $s \leq 3$. By the Isaacs Theorem [Is1] we have $s \geq 3$. Thus $s=3$. Since kernels of all characters from $\operatorname{Irr}\left(\varphi_{i}^{G}\right)$ do not contain $N$ and $|\operatorname{Irr}(G)-\operatorname{Irr}(G / N)| \leq 3$, it follows that

$$
\varphi_{i}^{G}=e_{i} \chi^{i}, \quad i=1,2,3, \quad \operatorname{Irr}(G)-\operatorname{Irr}(G / N)=\left\{\chi^{1}, \chi^{2}, \chi^{3}\right\} .
$$

Let $I_{G}\left(\varphi_{i}\right)$ be the inertia group of $\varphi_{i}$ in $G$. Set $\left|G: I_{G}\left(\varphi_{i}\right)\right|=t_{i}$. Then by Clifford's Theorem $\chi^{i}(1)=e_{i} t_{i} d_{i},|G: N|=e_{i}^{2} t_{i}, i=1,2,3$. Let $\pi$ be the set of all prime divisors of $|G: N|$. Then $\pi=\pi\left(e_{i} t_{i}\right), i=1,2,3$ (here $\pi(n)$ is the set of prime divisors of a positive integer $n$ ). As $k_{G}(N)=4$ it follows from Burnside's $\{p, q\}$-theorem that $N$ is simple. Take $p \in \pi$. If $\chi \in \operatorname{Irr}(G)$ and $p \nmid \chi(1)$, then $N \leq \operatorname{ker} \chi$ by the above. Denote by $G\left(p^{\prime}\right)$ the intersection of the kernels of all nonlinear $\chi \in \operatorname{Irr}(G)$ such that $p \nmid \chi(1)$. Obviously $N \leq G\left(p^{\prime}\right)$. Since $G\left(p^{\prime}\right)$ is $p$-nilpotent [Be] and $N$ is nonabelian simple, then $p \nmid|N|$. Thus $N$ is a $\pi^{\prime}$-Hall subgroup of $G$. By the Schur-Zassenhaus Theorem there exists in $G$ a $\pi$-Hall subgroup $H$. Since $N<G$, it follows that $H>\{1\}$. Take $x$, an element of prime order in $H$. Since $N$ is not nilpotent, there exists an element $y \in N-\{1\}$ such that $x y=y x$ ([Hu], Hauptsatz 4.8.7(a)). Since any $G$-conjugate of $x y$ is not contained in $H \cup N$, then

$$
k(H)+3=k(G / N)+3=k(G) \geq k(G / N)+k_{G}(N)-1+1=k(H)+4,
$$

which is a contradiction.

In Remark 1 we use the Tate Theorem (see [Is2, Theorem 6.31]). Let $A^{p}(G) / G^{\prime} \in \operatorname{Syl}_{p^{\prime}}\left(G / G^{\prime}\right), P \in \operatorname{Syl}_{p}(G)$. Obviously, $A^{p}(P)=P^{\prime}$. The Tate Theorem asserts that $P \cap A^{p}(G)=P^{\prime}$ implies $P \cap O^{p}(G)=O^{p}(P)=\{1\}$, where $O^{p}(G)$ is the unique minimal normal subgroup of $G$ such that $G / O^{p}(G)$ is a $p$-group. Assume that $N$ is a normal subgroup of $G$, and $P \cap N \leq P^{\prime}$. We shall prove that $N$ has normal $p$-complement. Without loss of generality we may assume that $G=P N$. Then $P^{\prime} N=A^{p}(G)$ and $P \cap P^{\prime} N=P^{\prime}$. By the Tate Theorem one obtains $P \cap O^{p}(G)=O^{p}(P)=\{1\}$ so $O^{p}(G)$ is a $p^{\prime}$-subgroup. Hence $G$, and so $N$, has a normal $p$-complement.

Remark 1. In the sequel we shall use the following assertion: If $N$ is a nontrivial normal subgroup of $G$ and $\left|\left\{\chi(1) \mid \chi \in \operatorname{Irr}_{1}(G)-\operatorname{Irr}(G / N)\right\}\right|=1$, then $N$ is solvable. There is an extension of elementary abelian group $N$ of order $2^{4}$ by $A_{5}$ which satisfies the above equality. Let us prove this assertion. Assume that $N$ is nonsolvable. Let $\{1\}<N_{1}<N$ and $N_{1}$ be normal in $G$. Since $\operatorname{Irr}_{1}(G / N) \subset \operatorname{Irr}_{1}\left(G / N_{1}\right)$ (this is due to the fact that the sum of the nonlinear irreducible characters of a nonabelian group is a faithful character), then

$$
\begin{gathered}
\operatorname{Irr}_{1}(G)-\operatorname{Irr}\left(G / N_{1}\right) \subset \operatorname{Irr}_{1}(G)-\operatorname{Irr}(G / N), \\
\operatorname{Irr}_{1}\left(G / N_{1}\right)-\operatorname{Irr}(G / N) \subset \operatorname{Irr}_{1}(G)-\operatorname{Irr}(G / N),
\end{gathered}
$$

and it suffices to prove our assertion in the case when $N$ is a minimal normal subgroup of $G$. Take a nonlinear $\lambda \in \operatorname{Irr}(N)$ and $\chi \in \operatorname{Irr}\left(\lambda^{G}\right)$. Let $p$ be a 
prime divisor of $\lambda(1)$. Then $p \mid \chi(1)$ by the Clifford Theorem. By reciprocity $N$ is not contained in $\operatorname{ker} \chi$, i.e. $\chi \in \operatorname{Irr}_{1}(G)-\operatorname{Irr}(G / N)$. Take $P \in \operatorname{Syl}_{p}(G)$. Then $P \cap N=P_{1} \in \operatorname{Syl}_{p}(N)$ and $P_{1}$ is not contained in $P^{\prime}$ according to the Tate Theorem (see text before this remark). Therefore there exists a linear character $\mu$ of $P$ such that $P_{1}$ is not contained in $\operatorname{ker} \mu$. Since $N^{\prime}=N$, it follows that $N \leq G^{\prime}$. Since $p \nmid \mu^{G}(1)$, there exists $\tau \in \operatorname{Irr}\left(\mu^{G}\right)$ such that $p \nmid \tau(1)$. By reciprocity $N$ is not contained in $\operatorname{ker} \tau$, so $\tau \in \operatorname{Irr}_{1}(G)-\operatorname{Irr}(G / N)$. Therefore $\tau(1)=\chi(1), p \mid \chi(1), p \nmid \tau(1)$, a contradiction. Thus, $N$ is solvable.

Remark 2. Sometimes in the sequel we will use the following proposition: Let $\chi=\lambda^{G} \in \operatorname{Irr}(G)$ be faithful, $H \leq G, \lambda \in \operatorname{Irr}(H)$, and $H / \operatorname{ker} \lambda$ an $S$-group. If $N$ is a minimal normal subgroup of $G$ and $N \leq H$, then $N$ is an $S$ group. This is true since $N$ is not contained in $\operatorname{ker} \lambda$, so $N_{1}$ is not contained in $\operatorname{ker} \lambda$ where $N_{1}$ is some simple direct factor of $N$. Then $N_{1} \cap \operatorname{ker} \lambda=\{1\}$, so the subnormal subgroup $N_{1} \operatorname{ker} \lambda / \operatorname{ker} \lambda\left(\cong N_{1}\right)$ of $S$-group $H / \operatorname{ker} \lambda$ is an $S$-group as well. Since $N$ is a direct product of groups isomorphic to $N_{1}$, it is an $S$-group, and our claim is proved.

Remark 2 is due to the referee.

Consider the following property of a group $G$ :

(*) Whenever $\chi, \tau \in \operatorname{Irr}(G)$ with $\operatorname{ker} \tau=\operatorname{ker} \chi$ and $\chi(1)<\tau(1)$, then $\chi$ is $S$-monomial.

We note that epimorphic images of $(*)$-groups are $(*)$-groups. Now the number of nonmonomial irreducible characters in $(*)$-groups is not bounded.

Theorem 2. Let $S$ be a set of simple groups containing groups of all prime orders. Then any (*)-group $G$ is an $S$-group.

Proof. Suppose that $G$ is a counterexample of minimal order. If $M, N$ are distinct minimal normal subgroups of $G$, then $M N / M(\cong N)$ as a normal subgroup of an $S$-group $G / M$ is an $S$-group $(G / M$ is an $S$-group by induction, so the claim follows from the Jordan-Holder Theorem). As $G / N$ is an $S$-group by induction, then $G$ is an $S$-group, a contradiction. Thus $G$ contains only one minimal normal subgroup $N$. By assumption $N$ is not an $S$-group. In particular $N$ is nonsolvable. Since $\bigcap \operatorname{ker} \tau=\{1\}$ (here $\tau$ runs over the set $\operatorname{Irr}(G))$, then a group with a unique minimal normal subgroup has a faithful irreducible character. Take in $\operatorname{Irr}(G)$ a faithful character $\chi$ of minimal degree.

Suppose that $\chi(1) \geq \tau(1)$ for all faithful $\tau \in \operatorname{Irr}(G)$. Then all faithful irreducible characters of $G$ have the same degree and $N$ is solvable by Remark 1 , a contradiction. Thus $\chi(1)<\tau(1)$ for some faithful $\tau \in \operatorname{Irr}(G)$, so $\chi$ is $S$-monomial by hypothesis. Therefore there exist $H \leq G$ and $\lambda \in \operatorname{Irr}(H)$ such that $H / \operatorname{ker} \lambda$ is an $S$-group and $\chi=\lambda^{G}$. Since $\chi$ is faithful and $G$ is not an $S$-group, then $H<G$. Take $\xi \in \operatorname{Irr}\left(\left(1_{H}\right)^{G}\right)$. Because $\left(1_{H}\right)^{G}$ is reducible, then $\xi(1)<\chi(1)$. Hence $N \leq \operatorname{ker} \xi$ and $N \leq \operatorname{ker}\left(\left(1_{H}\right)^{G}\right)=H_{G}=\bigcap_{x \in G} H^{x} \leq \dot{H}$. In view of Remark $2, N$ is an $S$-group, a contradiction.

Corollary 2.1. Let $S$ be the set of all groups of prime orders. $A$ group $G$ is solvable if and only if each $\chi \in \operatorname{Irr}(G)$ with $\chi(1)<b(G)$ is $S$-monomial.

Corollary 2.2. Let $\pi$ be a set of primes. A group $G$ is a $\pi$-group if and only if for each $\chi \in \operatorname{Irr}(G)$ there exist $H \leq G, \lambda \in \operatorname{Irr}(H)$ such that $H / \operatorname{ker} \lambda$ is $a$ $\pi$-group and $\chi=\lambda^{G}$. 
Let $X(G)$ be the set of all faithful irreducible characters of $G, Y(G)=\{\chi \in$ $X(G) \mid \chi$ is $S$-monomial $\}, V(G)=X(G)-Y(G)$.

Definition. A group $G$ is $M S_{k}$ if whenever $G / N$ is a monolith, then $|V(G / N)|$ $\leq k$ and $\tau(1) \leq \chi(1)$ for $\tau \in Y(G / N), \chi \in V(G / N)$.

We note that epimorphic images of $M S_{k}$-groups are $M S_{k}$-groups.

Theorem 3. Suppose that a set $S$ is such as in Theorem 2. If $G$ is an $M S_{3}$ group, then it is an $S$-group.

Proof. Assuming that $G$ is a minimal counterexample we see that $G$ contains only one minimal normal subgroup $N, G / N$ is an $S$-group and $N$ is not an $S$-group. Therefore $\operatorname{Irr}(G)$ contains a faithful character. Since $N$ is nonsolvable, there exist in $\operatorname{Irr}(G)$ at least four faithful characters by Lemma 1. Take in $\operatorname{Irr}(G)$ a faithful $S$-monomial character $\chi$ of minimal degree ( $\chi$ exists by condition). By definition $\chi(1) \leq \tau(1)$ for every faithful $\tau \in \operatorname{Irr}(G)$. Then there exist $H \leq G$ and $\lambda \in \operatorname{Irr}(H)$ such that $\chi=\lambda^{G}$ and $H / \operatorname{ker} \lambda$ is an $S$-group. Since $\left(1_{H}\right)^{G}$ is reducible, all its irreducible constituents $\mu$ satisfy $\mu(1)<\chi(1)$. Therefore $N \leq \operatorname{ker}\left(1_{H}\right)^{G} \leq H$, and $N$ is an $S$-group by Remark 2 , a contradiction.

In particular $M S_{0}$-groups are $S$-groups. A character $\chi \in \operatorname{Irr}(G)$ is said to be monolithic if $G / \operatorname{ker} \chi$ is a monolith. Note that $G$ is an $M S_{0}$-group if every monolithic character $\chi$ is $S$-monomial. In particular if every monolithic character $\chi$ of $G$ is monomial, then $G$ is solvable. This is a generalization of Taketa's Theorem.

In the same way we may prove the following

Proposition 4. Let $N>\{1\}$ be a normal subgroup of $G$. If all characters from $\operatorname{Irr}(G)-\operatorname{Irr}(G / N)$ are monomial, then $N$ is solvable. In particular, the intersection of the kernels of the nonmonomial irreducible characters of $G$ is solvable.

Proof. Suppose that $N$ is nonsolvable. Let $M$ be the last member of the derived series of $N$. By assumption $M^{\prime}=M>\{1\}$. Since $\operatorname{Irr}(G / N) \subseteq$ $\operatorname{Irr}(G / M)$, it follows that $\operatorname{Irr}(G)-\operatorname{Irr}(G / M) \subseteq \operatorname{Irr}(G)-\operatorname{Irr}(G / N)$, and it suffices to prove the proposition for $M$ instead of $N$. In view of Taketa's Theorem one has $M<G$. Since $\bigcap \operatorname{ker} \tau=\{1\}$ where $\tau$ runs over the set $\operatorname{Irr}_{1}(G)$, there is in $\operatorname{Irr}(G)-\operatorname{Irr}(G / M)$ a nonlinear character $\chi$ of minimal degree $(\chi$ is nonlinear in view of $\left.M=M^{\prime} \leq G^{\prime}\right)$. By condition there exist $H \leq G$ and $\lambda \in \operatorname{Lin}(H)$ such that $H / \operatorname{ker} \lambda$ is cyclic and $\chi=\lambda^{G}$. Take $\psi \in \operatorname{Irr}\left(\left(1_{H}\right)^{G}\right)$. Since $\chi(1)>1$, it follows that $H<G$ and $\left(1_{H}\right)^{G}$ is reducible. Hence $\psi(1)<\chi(1)$ so that $M \leq \operatorname{ker} \psi$ by the choice of $\chi$. Hence $M \leq \operatorname{ker}\left(\left(1_{H}\right)^{G}\right)=H_{G} \leq H$. Since $H / \operatorname{ker} \lambda$ is solvable and $M^{\prime}=M$, it follows that $M \leq \operatorname{ker} \lambda$. Therefore $M \leq \operatorname{ker} \chi-$ a contradiction with a choice of $\chi$. Let $D$ be the intersection of the kernels of the nonmonomial irreducible characters of $G$. Then all characters from $\operatorname{Irr}(G)-\operatorname{Irr}(G / D)$ are monomial. Hence, $D$ is solvable. ${ }^{1}$

\footnotetext{
${ }^{1}$ Analogously, if all characters from $\operatorname{Irr}(G)-\operatorname{Irr}(G / N)$ are $S$-monomial, then $N$ is an $S$ subgroup. In particular, the intersection of the kernels of the non- $S$-monomial irreducible characters of $G$ is an $S$-group. Instead of $M$ in the proof, we have to take $N^{S}$, the intersection of such normal subgroups $A$ in $N$ such that $N / A$ is an $S$-group.
} 
Conjecture 1. If all nonmonomial irreducible characters of a group $G$ have the same degree, then $G$ is solvable.

We do not know whether $G$ is solvable if it contains only one nonmonomial irreducible character.

Conjecture 2. Suppose that for every nonlinear $\chi \in \operatorname{Irr}(G)$ there exist $H<G$ (strict inclusion) and $\lambda \in \operatorname{Irr}(H)$ such that $\chi=\lambda^{G}$. Then $G$ is solvable.

Corollary 5. Suppose that $S$ is the set of groups of all prime orders. If all $\chi \in$ $\operatorname{Irr}(G)$ with $\chi(1)>3$ are $S$-monomial, then $G$ is solvable.

Proof. Take $\chi \in \operatorname{Irr}(G)$. Suppose that $\chi(1)<4$ and $G / \operatorname{ker} \chi$ is nonsolvable. Then from the classification of linear groups of degrees 2 and 3 it follows that there exists a normal subgroup $A / \operatorname{ker} \chi$ in $G / \operatorname{ker} \chi$ such that $G / A$ is one of the groups $\operatorname{PSL}(2,5), \operatorname{PSL}(2,7)$ [B1]. Take $\tau \in \operatorname{Irr}(G / A)$ such that $\tau(1)=4$ if $G / A=\operatorname{PSL}(2,5)$ and $\tau(1)=6$ if $G / A=\operatorname{PSL}(2,7)$. Since there is not a subgroup $H / A$ in $G / A$ such that $1<|G: H| \leq \tau(1)$, our condition does not hold for $G / A$ and so for $G$. Thus $G / \operatorname{ker} \chi$ is solvable for all $\chi \in \operatorname{Irr}(G)$ with $\chi(1)<4$.

Suppose that $G$ is a counterexample of minimal order. Then $G$ contains only one minimal normal subgroup $R, G / R$ is solvable and $R$ is not solvable. Take in $\operatorname{Irr}(G)$ a faithful character $\chi$ of minimal degree. By the above $\chi(1)>$ 3. Then there exist $H \leq G$ and $\lambda \in \operatorname{Irr}(H)$ such that $H / \operatorname{ker} \lambda$ is solvable and $\chi=\lambda^{G}$. Since for each irreducible constituent $\tau$ of $\left(1_{H}\right)^{G}$ one has $\tau(1)<$ $|G: H| \leq \chi(1)$, it follows that $R \leq \operatorname{ker} \tau$, so $R \leq \operatorname{ker}\left(1_{H}\right)^{G} \leq H$. Since $H / \operatorname{ker} \lambda$ is solvable and $R=R^{\prime}$, it follows that $R \leq \operatorname{ker} \lambda$, so $R \leq \operatorname{ker} \chi$, a contradiction.

It is impossible to replace in Corollary 5 the number 3 by 4 . In particular if all $\chi \in \operatorname{Irr}(G)$ with $\chi(1)>3$ are monomial, then $G$ is solvable.

Question. Classify all nonsolvable groups $G$ such that all $\chi \in \operatorname{Irr}(G)$ with $\chi(1)>4$ are monomial.

Denote by $p(G)$ the minimal prime divisor of $|G|$.

In the sequel we use the following known result ([Is2], Problem 3.4):

Lemma 6. Let $G$ be a nonabelian simple group, $p$ a prime divisor of $|G|$, $P \in \operatorname{Syl}_{p}(G)$. If $\chi \in \operatorname{Irr}(G)$ is faithful and $\chi(1)=p$, then $P$ is of order $p$.

Theorem 7. Suppose that for each irreducible character $\chi$ of $G$ there exist $H \leq$ $G, \lambda \in \operatorname{Irr}(H)$ such that $\lambda(1) \leq p(H)$ and $\lambda^{G}=\chi$. Then $G$ is solvable.

Proof. Assume that $G$ is a counterexample of minimal order. Then $G$ contains only one minimal normal subgroup $R, G / R$ is solvable and $R=F_{1} \times \cdots \times F_{s}$ where $F_{i}$ are isomorphic nonabelian simple groups. Hence $G$ has a faithful irreducible character. Take in $\operatorname{Irr}(G)$ a faithful character $\chi$ of minimal degree. By hypothesis there exist $H \leq G, \lambda \in \operatorname{Irr}(H)$ such that $\lambda(1) \leq p=p(H)$ and $\chi=\lambda^{G}$. To show that $R \leq H$, let us consider $\left(1_{H}\right)^{G}$. If $H=G$, then $R \leq H$. So suppose that $H<G$. Then $\left(1_{H}\right)^{G}$ is reducible. So all irreducible constituents of $\left(1_{H}\right)^{G}$ are not faithful (their degrees less than $\chi(1)$ ) and $R \leq \operatorname{ker}\left(1_{H}\right)^{G} \leq H$. Since $\chi=\lambda^{G}$ is faithful, $R=R^{\prime}$ is not contained in $\operatorname{ker} \lambda$. Hence $\lambda_{R}$ has no linear constituents. Therefore $\lambda(1)=p(H)$ and $\lambda_{R}$ is 
irreducible (Clifford). Therefore $p(H)|| R \mid$ and $p(H)=p(R)=p$. Moreover there exists $i \in\{1, \ldots, s\}$ such that the restriction of $\lambda$ on $F_{i}$ is irreducible. Let $P$ be a Sylow $p$-subgroup of $F_{i}$. Then $P$ is of order $p$ (Lemma 6) and $F_{i}$ has a normal $p$-complement by Burnside's normal $p$-complement theorem. Hence $R$ has a normal $p$-complement as well, contradicting the equality $R^{\prime}=$ $R$.

In particular if every irreducible character of $G$ is induced from a character of degree at most 2 , then $G$ is solvable.

Conjecture 3. If any irreducible character of a group $G$ is induced from a character of degree at most 3 , then $G$ is solvable. ${ }^{2}$

Conjecture 4. If all irreducible characters of $p^{\prime}$-degrees from $\operatorname{Irr}(G)$ are monomial, then $G$ is $p$-solvable, unless $p<5$.

Conjecture 5. If all irreducible characters of composite degrees are monomial, then $G$ is solvable.

Conjecture 6. Suppose that every $\chi \in \operatorname{Irr}(G)$ such that $\chi(1)$ is not a power of a fixed prime $p$ is monomial. Then $G$ is solvable.

Let $N$ be a normal subgroup of $G$. Set $c(N)=\mid\left\{\chi(1) \mid \chi \in \operatorname{Irr}_{1}(G), N\right.$ is not contained in $\operatorname{ker} \chi\} \mid$. If $c(N)=1$, then $N$ is solvable (Remark 1). Probably if $c(N)=2$, then $N$ is solvable too. If $N=G=A_{5}$, then $c(N)=3$.

\section{ACKNOWLEDGMENT}

I am indebted to the referee for useful comments and suggestions.

\section{REFERENCES}

[Be] Ya. G. Berkovich, Degrees of irreducible characters and normal p-complements, Proc. Amer. Math. Soc. 106 (1989), 33-35.

[B1] H. F. Blichfeldt, Finite collineation groups, Univ. of Chicago Press, Chicago, 1917.

[Hu] B. Huppert, Endliche Gruppen, Bd. 1, Springer, Berlin, 1967.

[Is1] I. M. Isaacs, Groups having at most three irreducible character degrees, Proc. Amer. Math. Soc. 21 (1969), 185-188.

[Is2] _ Character theory of finite groups, Academic Press, New York, 1976.

Department of Mathematics and Computer Science, University of Haifa, 31905 Haifa, ISRAEL

E-mail address: rsmaf01@haifa.uvm

\footnotetext{
${ }^{2}$ This conjecture is true. Moreover, if any irreducible character of a group $G$ is induced from a character of degree at most 4, then $G$ is solvable, unless $G / S(G) \cong A_{5}$; here $S(G)$ is the solvable radical of $G$ (see Ya. Berkovich, On the Taketa Theorem (to appear)).
} 